\title{
Using Adobe Flash Lite on mobile phones for psychological research: Reaction time measurement reliability and interdevice variability
}

\author{
STIAN ReImers \\ University College, London, England \\ AND \\ NeIL STeWART \\ University of Warwick, Coventry, England
}

\begin{abstract}
Mobile telephones have significant potential for use in psychological research, possessing unique characteristics - not least their ubiquity - that may make them useful tools for psychologists. We examined whether it is possible to measure reaction times (RTs) accurately using Adobe Flash Lite on mobile phones. We ran simple and choice RT experiments on two widely available mobile phones, a Nokia 6110 Navigator and a Sony Ericsson W810i, using a wireless application protocol (WAP) connection to access the Internet from the devices. RTs were compared within subjects with those obtained using a Linux-based millisecond-accurate measurement system. Results show that measured RTs were significantly longer on mobile devices, and that overall RTs and distribution of RTs varied across devices.
\end{abstract}

In recent years, one of the biggest sociotechnological developments has been the widespread adoption of mobile telephones throughout the western world. In 1998 in the United Kingdom, under 30\% of households owned a mobile phone (Office for National Statistics, 2008). By 2006 the proportion had risen to $80 \%$, and now in 2008 , the number of mobile phones exceeds the population. It is estimated that there are over two billion mobile phones globally (Central Intelligence Agency, 2008).

Although the primary use of a mobile phone is to make and receive calls and text messages, many phones now include other capabilities such as digital cameras, audio players, radios, games, and organizational tools such as alerts and planners. In the 10 years leading up to 2008 , it has become increasingly easy and popular to use a wireless application protocol (WAP) connection to access the Web from mobile devices. This has made it relatively simple to download games and applications across the Internet, check e-mail, and search on specially designed Web sites for information such as train times, traffic conditions, or such local facilities as restaurants or filling stations. Mobile Internet access has also been developed to tie in with more traditional PC-based access; for example, using WAP, it is now possible to place a bid on the auction site eBay (wap.ebay.com), or to update one's status on the popular social networking site Facebook (m.facebook .com) from a mobile phone costing under $\$ 100$. Thus, at the time of writing, it appears that Internet use on mobile phones is becoming mainstream.

\section{Existing Research Using Mobile Devices}

A WAP-enabled mobile phone is essentially a small, cheap, handheld computer that can send and receive data wirelessly across the Internet. Put in these terms, mobile phones may appear attractive as devices for running psychology experiments. However-perhaps unsurprisingly, given the speed at which mobile phone capabilities have developed - there has so far only been limited psychological research using mobile devices. Shephard, Kho, Chen, and Kosslyn (2006) developed a system, MiniCog, for administering cognitive tests primarily to astronauts, but also "in the field" generally, using a personal digital assistant (PDA). This system was capable of measuring reaction times (RTs) on cognitive tasks, and the authors noted that the RTs should be accurate to around $10 \mathrm{msec}$, although they indicated that they had not conducted any systematic tests of RT accuracy. MiniCog was also designed as a package that would be downloaded, and data uploaded, via a host computer, rather than by direct contact with a server across the Internet.

A different approach to using mobile devices for psychological research was taken by Hogarth and colleagues (e.g., Hogarth, Portell, \& Cuxart, 2007), who used mobile phone short message service (SMS) text messages to implement

S. Reimers, s.reimers@ucl.ac.uk 
an experience sampling method of risk perception measurement. They sent text messages to participants at random times during the day. When participants received the message they had to make a note of what they were doing, and answer questions about the potential risks that the activity they were engaging in entailed. We presume that-since two of the questions had an open-ended format - they did not submit their responses by mobile phone.

Text messaging has also been used in classroom experiments. Cheung (2008) used a sophisticated system for recording, processing, and responding to SMS text messages from students in a class. He used the system to run a version of the ultimatum and public goods games. Thus, half the students in the ultimatum game setup sent a text message with the amount they would offer; the other half sent a text message with the minimum amount they would accept. Proposers and responders were randomly paired, and each received a text message informing them whether agreement had been reached or not. Summary statistics from the game were also generated using a macro, and the results were displayed for the class to see.

\section{Advantages and Disadvantages of Mobile Phones in Psychological Research}

The three examples above show some of the major advantages of using mobile devices for psychological research. Participants can undergo testing "in the field" in more naturalistic situations, and with little notice or effort. The ubiquity of mobile phones means that large numbers of people can participate in an experiment without the need to allocate a device to collect responses.

However, we suggest that there are additional reasons that using mobile devices might be of benefit to psychologists. Much of the literature on the advantages and disadvantages of Web-based testing can also be applied to running experiments on mobile devices. Thus, mobile phone testing allows one to test participants who are not physically present in the lab, and to reach a broader demographic than those who normally participate in lab-based research. In particular, it may be easier to run experiments with certain subgroups of the population, such as those with limited mobility, those living far from a researcher's institution, and others who would be unwilling to go to the effort of making an appointment and traveling to the laboratory for testing. It also gives people more flexibility in participating and allows them to participate from the familiar surroundings of their own homes.

As well as the potential advantages that phone-based testing shares with traditional computer-based Web testing, mobile devices have a number of other advantages. As we saw in the examples of Shephard et al. (2006) and Hogarth et al. (2007), they allow testing to be done "in the field," where it would be harder to test using a traditional computer-based setup. Shephard et al. used the extreme example of testing in space, but also suggested that there may be down-to-earth scenarios that could be explored using mobile devices. Examples might include testing people in extreme situations, such as before and after a sporting event, a parachute jump, or a presentation to hundreds of people; tracking the effects of, say, fatigue in drivers, people doing shift work, or people with high-stress jobs such as air traffic control or city trading; examining the effects of alcohol, nicotine, or illegal drugs on cognition; or examining how people's quotidian activities affect cognitive or emotional states.

Mobile phones also have the advantage that they tend to be switched on and carried by their owners most of the time. It would therefore be possible to do as Hogarth et al. (2007) did and contact people, or ask them to respond at a specific time. Thus, the effect of circadian rhythms could easily be investigated. Alternatively, memory stimuli with interitem spacings of hours or days could be delivered using mobile phones.

Finally, Cheung's (2008) setup could conceivably be extended, using a Bluetooth or WAP connection instead of SMS messaging to allow lecturers to run experiments in many areas of cognition, presenting visual stimuli and recording RTs, showing students how particular "classic" experiments were implemented and the types of results obtained. Whole practical classes could be run without the need for a large number of computers.

Clearly, mobile phones are not a panacea, and there are also significant potential drawbacks that might need to be overcome. These include demographics skewed toward the younger and more affluent members of society, particularly for experiments that require WAP, Java, or Flash. There are also problems of limited geographical network coverage: Participants might miss a message or request, or may fail to save their data, if they are out of signal range. Responses may also be interrupted by incoming calls or messages. The cost of sending and receiving data using WAP can be very high, largely precluding the use of audio and video stimuli. The screen size is relatively small, keys are also small and fiddly, and free-text responses are, at best, difficult to give.

In summary, although there are several potential problems to overcome for some types of phone-based testing, there appear to be enough potential advantages to take the notion of phone-based testing seriously.

\section{RT Experiments on Mobile Devices}

Having discussed the use of mobile devices generally, we now focus specifically on running experiments that measure millisecond RTs. We know of three broad ways of running RT experiments on mobile devices. One is to download and install a program that runs on the phone's operating system. For example, there are many applications that have been developed for the Symbian operating system found on many brands of mobile device. This is broadly equivalent to downloading and installing an executable file on a PC. The advantages of this technique are that the program, once installed, has access to a wide range of the device's functionalities, such as establishing Web connections, sending text messages, and operating the camera. A major disadvantage is that people are often reluctant to install software that is so unconstrained. Furthermore, a piece of software will only run on phones that use the operating system for which it was designed.

A second way would be to install a freestanding application written in Java that has limited access to the phone's 
data and processes. The majority of mobile phone games are written in Java, and users generally download an application and respond to a prompt asking whether they wish to install the program, and the application is then saved locally on the user's device. The functionality of Java is sandboxed, so that an application cannot access other information on the device, or use all of its functionality. Thus, Java trades off perceived security threats with decreased functionality.

Third and finally - and this is the focus of our attention here-it is possible to run experiments using Flash Lite. Flash Lite differs from Java because many mobile devices are set up to run Flash Lite applications automatically after download without asking users whether they want to run the code. This makes the experience of completing Flash Lite experiments more ergonomic for the user than Java, and also means that on many devices, Flash Lite applications can be embedded in HTML code, allowing developers to use a combination of Flash and HTML in their applications. Potential drawbacks of Flash Lite are the very restricted access programs have to other information stored, and processes running on the phone; Flash Lite 1.1 cannot use Bluetooth, for example. Also, running in a browser may lead to a decrease in performance.

\section{Adobe Flash and Adobe Flash Lite}

Adobe's range of Flash products have been used extensively to develop and deliver games, advertisements, and other interactive content over the Web, and have been used for running RT-based psychology experiments (see Reimers \& Stewart, 2007, for an introduction, and Reimers, 2007, and Reimers \& Maylor, 2005, for examples). In the past few years, one of the major developments in the use of Flash has been the release of Flash Lite, a pared-down version of Flash designed to be run on mobile devices. Flash Lite is included in a number of retail mobile devices. Strategy Analytics (Robinson, 2008) reports that between 2003 and 2007, over 300 million Flash Lite-enabled devices were shipped, and this number is predicted to rise to 1.4 billion by the end of 2010. A list of current devices supporting Flash Lite can be found at www.adobe.com/ mobile/supported_devices/handsets.html.

One advantage of Flash Lite is its similarity to standard Flash. This means that researchers who are used to implementing Web experiments in Flash will find it relatively easy to write experiments in Flash Lite. Also, an experiment has to be written only once; although appearances may differ slightly on different devices, all are capable of reading and executing Flash Lite files.

We therefore set out to investigate whether it would be viable to run RT psychology experiments using Flash Lite and, in particular, the extent to which currently available devices could record RTs consistently and accurately.

\section{The Present Study}

In the present study, we extended the research we conducted on measuring RTs using Flash over the Web (Reimers \& Stewart, 2007) to measuring RTs using Flash Lite and WAP on two widely available mobile phones. We had two aims. The first was to examine generally whether RT measurement is accurate enough on currently available mobile devices for RT-based testing to be worth considering at all. For example, if RTs measured on mobile phones were hundreds of milliseconds longer than those obtained under accurate measurement, or were quantized to the nearest $500 \mathrm{msec}$, testing using mobiles would be unviable. The second aim was to examine the extent to which RT measurement accuracy varies across devices. This is important, because if there is significant variation it will be necessary to ensure that differences in RT due to hardware are accounted for, and are not assumed to be psychological differences.

To investigate this, we took two mobile phones, one very modern, one somewhat older, and compared — within subjects-RTs measured on each of them with an accurate baseline. Our baseline condition, which is known to measure RTs with millisecond accuracy, was programmed in C, and used the same setup described in Stewart (2006a, 2006b). In the other two conditions, a single Flash Lite 1.1 swf (Shockwave Flash) file was run on a Nokia 6110 and a Sony Ericsson W810i. The experiment was designed as more of a field test than as a calibration: We tested the same participants in the three different conditions and compared RTs. This does conflate technological variables, such as the measurement accuracy of a particular phone, with ergonomic variables, such as using thumbs on a mobile phone keypad versus index fingers on a buttonbox, and motivation issues: The baseline setup looked much more like a "proper" psychology experiment than a quiet room with a mobile phone would have, so participants may have been motivated to try harder. It is also possible that the smaller size of the stimuli on the screen made the mobile condition harder. Thus, the results of this experiment should not be interpreted as being the result of only the physical characteristics of the different devices, but rather the overall differences in RTs one might expect if one ran participants in the different conditions.

\section{METHOD}

\section{Participants}

Participants were 22 undergraduate psychology students at the University of Warwick. All participated for course credit; there was also a $£ 20$ (US\$40) prize for the best performance across all conditions.

\section{Design and Procedure}

There were three within-subjects conditions: one baseline, using the Linux setup described below, and two others, Nokia and Sony, which used the same Flash code, designed to appear as similar as possible to the baseline condition. The order in which participants used the different devices was counterbalanced.

The system used for the desktop-computer-based experiments (baseline) was a Tranquil T2, a silent, low-cost PC. Stimuli were presented on a Viewsonic VX924 19-in. LCD display. Responses were collected using a custom-made parallel-port button box with two buttons, one for the left index finger and one for the right index finger. The Nokia condition used the 6110 Navigator, with a $320 \times$ 240 pixel screen, in standard U.K. configuration, connected to the Vodafone network. The 6110 is, at the time of writing, a high-end $3 \mathrm{G}$ phone, with built-in GPS, and it retails for around $£ 200$ (US\$400). 
The Sony condition used the Sony Ericsson W810i in standard U.K. configuration, with a $220 \times 176$ pixel screen, connected to the $\mathrm{O} 2$ network. The W810i is a midmarket phone, released in early 2006, which currently retails for around $£ 75$ (US\$150).

In the baseline condition, the $\mathrm{C}$ code was executed from the command line and the experiment window filled the screen. In the Nokia condition, the Flash was downloaded using WAP, and was run full-screen from a freestanding version of Flash Player (since Flash Player had not been installed as a browser plug-in); in the Sony condition, the experiment was downloaded using WAP, and displayed in the device's browser, which was set to full screen. In both the phone conditions, the Flash Lite code was downloaded in its entirety before the experiment began. Thus, until data were saved at the end of the experiment, the cellular network was not required.

Participants completed two 30-trial tasks, choice RT (CRT) and simple RT (SRT), always in that order. In all conditions, participants sat alone in a quiet room. At the start of each condition, printed instructions were given and were summarized on the monitor or device screen. At the start of a trial, a "+" crosshair appeared on the screen, followed by a variable delay of between approximately 1,500 and 3,000 msec (see below). Following the delay, in the CRT task, the crosshair was replaced by a red or green rectangle, which remained on the screen until a response was made. Immediate feedback ("correct!" or "wrong!" and the RT in milliseconds) was given, lasting $1,000 \mathrm{msec}$ before the next trial began. All trials were independent: On each trial there was an equal (.5) chance of the rectangle being red or green. Response key mapping was counterbalanced: Half the participants pressed the left key if the rectangle was green, and the other half pressed if it was red. Response key mapping was the same for a given participant in all three conditions. In the SRT task, the crosshair was replaced by a blue rectangle, which remained on the screen until a response was given. Participants could respond using either key from the CRT task, and used the same finger and button throughout the SRT task. The RT in milliseconds was displayed as feedback for 1,000 msec before the next trial began. In the baseline condition, participants were instructed to use their left and right index fingers to respond. In the phone conditions, participants were allowed to choose the digits with which they responded (as would be the case with unsupervised remote participants), but were informed that cradling the phone and using left and right thumbs to respond might be most comfortable. (In earlier work, we had found that most participants naturally ended up responding in this manner, and we did not want to add noise to our data from people changing hand positions, and hence possibly altering RTs, part of the way through the experiment.)

At the end of a set of 30 CRT or SRT trials, all the data from all 30 trials were saved to disk (in the baseline condition) or transmitted using WAP to a Perl script on our server, which handled saving the data (in the mobile conditions). Data in the mobile conditions were not saved on the phone itself.

\section{Implementation}

Flash (Nokia, Sony). A $220 \times 176$ pixel Flash Lite 1.1 movie was created using Adobe Flash CS3. Because Flash Lite 1.1 has very restricted capabilities relative to normal Flash and Flash Lite 2.0 , the implementation was slightly different from that in Reimers and Stewart (2007). To create a variable stimulus onset delay, we created 16 frames, which were played in sequence. With each frame there was a probability of jumping to a later frame in which the stimulus appeared. Thus, in Frame 1 there was a 1/16 chance of a jump to the stimulus display frame; in Frame 2 there was a 1/15 chance, and so on up to Frame 15, where there was a 1/2 chance of jumping, and Frame 16, where there was a 1/1 (i.e., certain) chance of jumping. This would, in theory, give a flat distribution of 16 different delays of 1,500 $\mathrm{msec}, 1,600 \mathrm{msec}, 1,700 \mathrm{msec}$, and so on, up to $3,000 \mathrm{msec}$, assuming that the theoretical frame rate had been achieved.

We used pilot data to generate delays in the baseline condition that had approximately the same distribution as those actually seen on the Sony Ericsson, with the shortest of the 16 delay groups set at $1,526 \mathrm{msec}$ and the longest set at 3,291 msec. We also logged the actual delays on the Sony Ericsson during the experiment, which came out slightly longer than in the pilot, with the shortest delay category being $1,563 \mathrm{msec}$ and the longest delay being 3,369 msec. The Nokia, although running the same code as the Sony, had shorter delays, with a shortest delay category of 1,421 msec and a longest of $3,061 \mathrm{msec}$.

In the stimulus display frame, the movie playhead was stopped, one of two stimuli-a red or a green rectangle - was set visible, the other invisible, and a timer was polled using the getTimer () method. An on-key-down event listener waited for the "1" or " 3 " button to be pressed, after which the timer was polled again, and the difference was recorded.

C (baseline condition). The X Windows System was used to run a C program, which displayed stimuli (Stewart, 2006a). Responses were recorded on a parallel port buttonbox (Stewart, 2006b). Following Finney (2001), the program was scheduled to the highest priority (SCHED FIFO) so it was not interrupted, had its memory locked (with a call to mlockall () ) to prevent swapping to disk, and so that timings were measured (using the gettimeofday ()) to the nearest microsecond. RTs were measured from the end of the vertical retrace upon which the stimulus was drawn to the first detection of a close of a button on the parallel port buttonbox.

\section{RESULTS}

During testing, the remote server from which the Flash versions of the experiment were being accessed, and to which data were being saved, crashed temporarily. This meant that data from participants who were part of the way through either of the Flash tasks were lost, and the participants in one time slot were excused from the Flash conditions. (We note here that the crash was due to our dubious choice of Internet service provider, rather than to failures of Flash or the mobile phone network.) We were left with 20 participants for whom we had data for all three SRT conditions.

In the CRT condition we had 15 participants. The server problems were compounded by (we suspect) the fact that, in the Nokia condition - in which the experiment was run using the standalone Flash Player - the data occasionally expired before the Web was accessed at the end of the SRT stage. For both SRT and CRT conditions, we only used participants for whom we had data for all three device cells.

The group means and standard deviations ( $S D \mathrm{~s})$ for the SRT and CRT conditions, along with error rates for CRT responses, are given in Tables 1 and 2. Only RTs between 100 and $1,000 \mathrm{msec}$ are included in the analysis (SRT, $98.2 \%$ of baseline trials, $98.7 \%$ of Nokia trials, and $98.0 \%$ of Sony trials; CRT, $99.6 \%$ of baseline trials, $98.9 \%$ of Nokia trials, and $98.9 \%$ of Sony trials).

Table 1

Mean Effects (in Milliseconds) of Testing Conditions on Average and Variability of SRT

\begin{tabular}{lcccccc}
\hline \multirow{2}{*}{ Condition } & \multicolumn{2}{c}{ Average } & & \multicolumn{3}{c}{ Variability } \\
\cline { 2 - 3 } \cline { 5 - 6 } Median & & $S D$ & Interquartile & Full \\
Range & Range \\
\hline Baseline & 214.4 & 202.6 & & 53.2 & 34.8 & 268.0 \\
Nokia & 266.9 & 242.0 & & 75.3 & 60.6 & 330.3 \\
Sony & 286.0 & 276.0 & & 56.9 & 45.3 & 272.7 \\
\hline
\end{tabular}


Table 2

Mean Effects (in Milliseconds) of Testing Conditions

on Average and Variability of CRT, and Error Rate

\begin{tabular}{|c|c|c|c|c|c|c|}
\hline \multirow[b]{3}{*}{ Condition } & \multirow{2}{*}{\multicolumn{2}{|c|}{ Average }} & \multicolumn{3}{|c|}{ Variability } & \multirow[b]{3}{*}{ Error Rate \% } \\
\hline & & & \multirow[b]{2}{*}{$S D$} & \multirow{2}{*}{$\begin{array}{c}\text { Interquartile } \\
\text { Range }\end{array}$} & \multirow{2}{*}{$\begin{array}{c}\text { Full } \\
\text { Range }\end{array}$} & \\
\hline & $M$ & Median & & & & \\
\hline Baseline & 339.7 & 329.2 & 74.7 & 78.3 & 342.1 & 4.0 \\
\hline Nokia & 383.4 & 366.5 & 86.6 & 88.9 & 366.6 & 4.7 \\
\hline Sony & 411.8 & 405.2 & 73.1 & 83.2 & 307.1 & 5.6 \\
\hline
\end{tabular}

To test the significance of any differences in RT and $S D$ for the SRT data, we ran repeated measures ANOVAs with condition (baseline, Nokia, Sony) as a within-subjects variable. (With 24 counterbalancing permutations, it was not possible to include counterbalancing as a betweensubjects variable.) There was a significant effect of condition on mean $\mathrm{RTs}[F(2,38)=79.1, p<.001]$ and median $\operatorname{RTs}[F(2,38)=123.8, p<.001]$, and variable effects on measures of variability: $\operatorname{SD}[F(2,38)=3.38, p=.048]$, interquartile range $[F(2,38)=6.62, p=.003]$, and full range $[F(2,38)=1.01]$.

We conducted an identical analysis for the CRT data. There was a significant effect of condition on mean RT $[F(2,28)=28.7, p<.001]$ and median $\mathrm{RT}[F(2,28)=$ $43.9, p<.001]$, but not on any of the measures of variability: $\operatorname{SD}[F(2,28)=1.04]$, interquartile range $[F(2,28)=$ $0.81]$, or full range $[F(2,28)=0.62]$.

We next examined responses for evidence of quantizing or other irregularities in the distribution of RTs. As in Reimers and Stewart (2007), we plotted a cumulative frequency graph of RTs in the three conditions (Figure 1).

The baseline and Nokia RTs followed the expected smooth sigmoid shape that reflects an underlying approximately normal distribution. In contrast, the Sony RTs showed approximately 20-msec plateaux around 250,370, and $480 \mathrm{msec}$, followed by an increased gradient shortly after. This suggests that the Sony delays processing of a response at certain times.

\section{DISCUSSION}

Our investigation showed that RTs measured on two mobile phones were, on average, $60-80 \mathrm{msec}$ longer than those measured using a millisecond-accurate Linux system. RTs were also device-specific: Those measured on the Nokia Navigator were $20-30 \mathrm{msec}$ shorter than were those on the Sony Ericsson W810i. In addition, RTs on the Sony Ericsson appeared to be substantially quantized.

\section{Cause of Differences}

There was a clear difference in measured RTs between the two phones, and between each phone and the baseline condition. We ran this experiment as a field-test comparison of devices rather than as a calibration, and so did not attempt to distinguish between the technical performance of the phones and the ergonomic effects of using thumbs to respond, small display size, posture, and other differences between computer and phones. However, the fact that RTs were so much faster on the more powerful Nokia than on the less powerful Sony suggests that technical performance was an issue.

The quantizing effects on the Sony but not on the Nokia also suggest performance limitations. The quantizing appears to occur at around the same rate as the Flash frame rate (and hence screen-update rate), which suggests that it might be the result of the processor being overloaded when running screen updates and other housekeeping activities at the start of each frame. If this is the case, it appears that the Nokia is powerful enough to avoid any quantizing of RTs upon entering a frame. Whether these effects are due to differences in processor power, or to running Flash inside a browser on the Sony, is not clear. Although the quantizing effects are not desirable, averaging across many trials would largely remove any effects. Clearly, the quantizing could be specific to the way in which we programmed the experiment, and there may be other implementations that avoid this problem. However, it is the fact that the quantizing occurred on only one of the two phones running the same code that suggests that RTs recorded on different devices may exhibit qualitative as well as quantitative differences.

\section{CONCLUSION}

\section{Recommendations for Mobile RT Research}

Overall, we think that mobile phones may be used for running RT-based research. Mean RTs were longer, but RT $S D$ s were only slightly increased, suggesting that, on the Nokia at least, it would be relatively easy to detect within-subjects changes to RTs, or-controlling for the device used-differences across between-subjects conditions. It is also clear that mobile devices are becoming increasingly powerful, and this may well lead to the attenuation of differences in RTs measured across devices. Finally, some of these effects may be specific to using Flash Lite-embedded in a browser, in the case of the Sony condition - to run experiments. It would be interesting to compare RTs measured using Flash with those measured using Java.

One key implication of these results for running experiments on mobile devices is that there is a risk of confounding device capabilities with variables that are under investigation. For example, if older people tend to own less recent mobile phones, any apparent age-related decline in RT may in fact be caused by the device rather than the person. However, there are several ways around the 


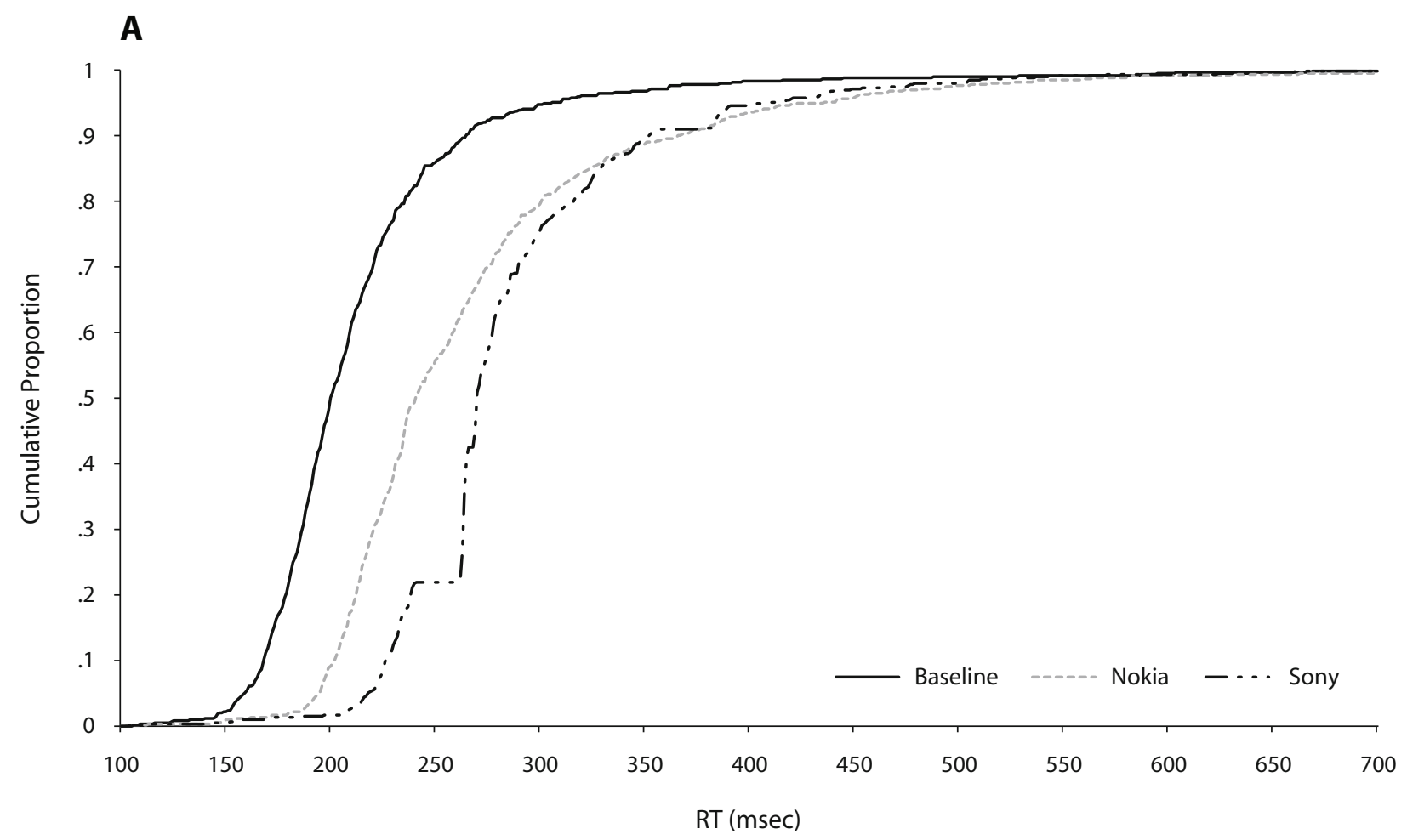

B

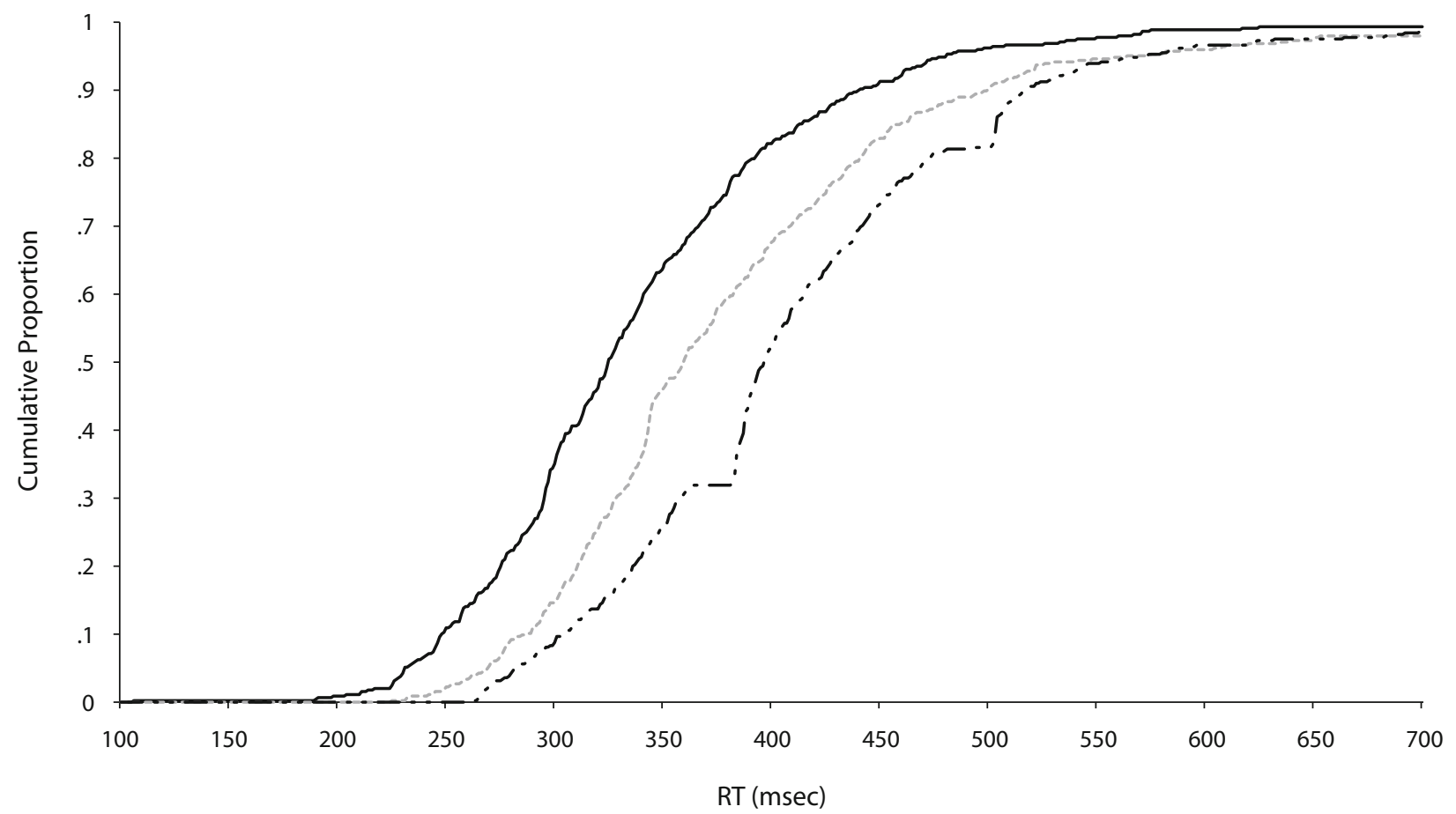

Figure 1. Cumulative frequency of trials as a function of RT (msec) for millisecond-accurate Linux baseline, and the two mobile devices. (A) SRT. (B) CRT. 
issue. One is to note the device used to run an experiment, which in Flash Lite 1.1 and above can be done using the getDevice () method, and include it as a variable in any analysis. A second is to use a within-subjects design, so that absolute effects disappear. A final, albeit crude, option might be to run an internal calibration, measuring how long it takes to execute a particular piece of looped code on a given device, to gain a measure of the processing resources it has available. If experiments and analyses are designed to take into account differences in device performance and the slight distortions to RT means and $S D$ s, we see no reason why mobile phones should not be used in RT-based psychological research.

\section{AUTHOR NOTE}

We thank Sarah Reimers for trusting us with the Nokia. This research and S.R. were supported by a fellowship from the ESRC Centre for Economic Learning and Social Evolution (ELSE). N.S. was supported by ESRC Grants ESRC RES-062-23-0952, ESRC RES-000-22-2459, and ESRC RES-000-23-1372. Correspondence concerning this article should be addressed to S. Reimers, Department of Psychology, University College, London, WC1H 0AP, England (e-mail: s.reimers@ucl.ac.uk).

\section{REFERENCES}

Central Intelligence Agency (2008). The CIA world factbook, rank order, telephones, mobile cellular. Retrieved February 2, 2008, from https://www.cia.gov/library/publications/the-world-factbook/ rankorder/2151rank.html.

Cheung, S. L. (2008). Using mobile phone messaging as a response medium in classroom experiments. Journal of Economic Education, 39, 51-67.

FinNey, S. A. (2001). Real-time data collection in Linux: A case study. Behavior Research Methods, Instruments, \& Computers, 33, 167-173.

Hogarth, R. M., Portell, M., \& Cuxart, A. (2007). What risks do people perceive in everyday life? A perspective gained from the experience sampling method (ESM). Risk Analysis, 27, 1427-1439.

Office for National Statistics (2008). Consumer durables: Consumer durables ownership increases. Retrieved February 2, 2008, from www.statistics.gov.uk/CCI/nugget.asp? ID $=868$.

ReIMERs, S. (2007). The BBC Internet study: General methodology. Archives of Sexual Behavior, 36, 147-161.

ReImers, S., \& MaYlor, E. A. (2005). Task switching across the life span: Effects of age on general and specific switch costs. Developmental Psychology, 41, 661-671.

Reimers, S., \& Stewart, N. (2007). Adobe Flash as a medium for online experimentation: A test of reaction time measurement capabilities. Behavior Research Methods, 39, 365-370.

Robinson, S. (2008). Flash-enabled handset forecast: $91 \%$ global growth in 2007 as Flash Lite gains momentum outside Japan. Abstract retrieved February 2, 2008, from www.strategyanalytics.net/default .aspx? $\bmod =$ ReportAbstractViewer\&a0 $=3727$.

Shephard, J. M., Kho, S., Chen, J., \& Kosslyn, S. M. (2006). MiniCog: A method for administering psychological tests and experiments on a handheld personal digital assistant. Behavior Research Methods, 38, 648-655.

STEWART, N. (2006a). Millisecond accuracy video display using OpenGL under Linux. Behavior Research Methods, 38, 142-145.

STEWART, N. (2006b). A PC parallel port button box provides millisecond response time accuracy under Linux. Behavior Research Methods, 38, 170-173.

(Manuscript received February 8, 2008; revision accepted for publication June 18, 2008.) 